\title{
Multiple superionic states in helium-water compounds
}

\author{
Cong Liu, ${ }^{1}$ Hao Gao, ${ }^{1}$ Yong Wang, ${ }^{1}$ Richard J. Needs, ${ }^{2}$ Chris J. \\ Pickard, ${ }^{3,4}$ Jian Sun, ${ }^{1, *}$ Hui-Tian Wang, ${ }^{1}{ }^{\dagger}$ and Dingyu Xing ${ }^{1}$ \\ ${ }^{1}$ National Laboratory of Solid State Microstructures, \\ School of Physics and Collaborative Innovation Center of Advanced Microstructures, \\ Nanjing University, Nanjing, 210093, P. R. China \\ ${ }^{2}$ Theory of Condensed Matter Group, Cavendish Laboratory, \\ J J Thomson Avenue, Cambridge CB3 OHE, UK \\ ${ }^{3}$ Department of Materials Science 86 Metallurgy, University of Cambridge, \\ 27 Charles Babbage Road, Cambridge CB3 OFS, UK \\ ${ }^{4}$ Advanced Institute for Materials Research, Tohoku University 2-1-1 Katahira, Aoba, Sendai, 980-8577, Japan
}

(Dated: May 23, 2019)

\begin{abstract}
Superionic states are phases of matter that can simultaneously exhibit some of the properties of a liquid and of a solid. For example, in superionic ice, hydrogen atoms can move freely while oxygen atoms are fixed in their sublattice. "Superionicity" has attracted much attention both in fundamental science and applications. Helium is the most inert element in nature and it is generally considered to be unreactive. Here we use $a b$ initio calculations to show that $\mathrm{He}$ and $\mathrm{H}_{2} \mathrm{O}$ can form stable compounds within a large pressure range which can exist even close to ambient pressure. Surprisingly, we find that they can form two previously unknown types of superionic states. In the first of these phases the helium atoms exhibit liquid behavior within a fixed ice-lattice framework. In the second of these phases, both helium and hydrogen atoms move in a liquid-like fashion within a fixed oxygen sublattice. Because the $\mathrm{He}-\mathrm{O}$ interaction is weaker than the $\mathrm{H}-\mathrm{O}$ interaction, the helium atoms in these superionic states have larger diffusion coefficients and lower "melting" temperatures than that of hydrogen, although helium is heavier than hydrogen. The insertion of helium atoms substantially decreases the pressure at which superionic states may be formed, compared to those in pure ice.
\end{abstract}

The pioneering work of Demontis et al. [1] and Cavazzoni et al. [2] led to studies of the "hot ice" layer between the rocky core and gaseous atmosphere in Uranus and Neptune, and proposed a surprising new phase between the solid and the fluid. This is a "superionic" state which is characterized by a fixed sublattice of oxygen or nitrogen atoms, while the hydrogen atoms diffuse almost freely throughout the sublattice. Since then, many theoretical and experimental studies have been devoted to investigating this important state of matter [3-20]. For instance, experimental electrical conductivity measurements [3, 4] and Raman signals [5] of water under compression indicate the existence of superionic states. The superionic water seems to be adjacent to the ordered ice VII and ice X, and significant ionic conductivity is found. [10] Very recently, Millot et al. [19, 20] investigated water ice under shock compression conditions and showed that ice melts near $5000 \mathrm{~K}$ at $190 \mathrm{GPa}$, which is close to the conditions found in the interiors of planets, and may have connections to superionic states.

Theoretical simulations were used to investigate the phase diagram of superionic water under conditions that may be found in planetary interiors. [7, 8] Recent simulations suggested that the oxygen sublattice in superionic water experiences a complicated evolution under

\footnotetext{
* E-mail: jiansun@nju.edu.cn

$\dagger$ E-mail: htwang@nju.edu.cn
}

compression, with phases such as bcc, fcc, closed-packed and $P 2_{1} / c$, etc. $[11,12]$. To study these transformations, thermodynamic potentials and entropies for superionic water were calculated. [14] Phases of superionic water (VII' and $\mathrm{VII}^{\prime \prime}$ ) were proposed, and $[15,18]$ superionic states in ammonia and ammonia-water mixtures have also been investigated $[9,13,16,17]$. For instance, Bethkenhagen et al. [13, 16] studied equations of state of mixtures of water, ammonia, and methane, and developed a new model of the interior structure of Uranus. Jiang et al. [17] found an ionic phase in ammonia dihydrate, which transforms into a superionic phase under high temperature and pressure. Due to the chemical similarity of hydrogen and lithium, the mechanism of hydrogen diffusion in the superionic state has been used to explore ionic conductivity in electrodes and electrolyte materials, especially in lithium-based battery materials [21-23]. It seems that superionic states also have connections with the recently discoverd copper-based thermolectric materials [24, 25].

Knowledge of the behavior of helium at different temperatures and pressures is key to understanding the nature of celestial bodies, as it is the second most abundant element in the universe after hydrogen and it is also present in large quantities in the atmospheres of giant planets such as Uranus and Neptune. Helium, as the most inert element in the periodic table, is generally considered to be unreactive due to its stable closed-shell electronic configuration with filled $s$ valence orbitals. Pres- 
sure is often used to explore new states of matter. Even a material as inert as helium can react with some elements and compounds under high pressure. For instance, a mixture with other inert gases [26, 27], such as nitrogen $[28,29]$, reacts with sodium [30], iron [31] or iron peroxide [32], alkali oxides or sulfide compounds [33] and alkaline earth fluorides [34]. The addition of helium can also reduce the pressure at which polymerization of nitrogen may occur. [35]. It has been reported that helium can react with water to form $\mathrm{He}\left(\mathrm{H}_{2} \mathrm{O}\right)_{2}$ at very high pressures (297 GPa) [36]. Teeratchanan and Hermann [37] recently found that helium can fill the voids in ice clathrates at very low pressures.

However, there is still a gap between the pressure of a few GPa and that of $300 \mathrm{GPa}$ which has not been explored in the helium-water system, although this turns out to be the pressure range in which superionic states can emerge in ice. On the other hand, helium compounds are not easily formed and superionicity in helium-based structures has not so far been reported. Moreover, the diffusive motions of helium in these compounds are also unknown. Beyond the "hot ice" layer in Uranus and Neptune, helium is gaseous, which motivates exploration of the relationship between helium and water at low and medium pressures.

\section{Results}

Crystal structures. We have used first-principles crystal-structure searching techniques to determine the most stable phases of various compositions of $\mathrm{He}-\mathrm{H}_{2} \mathrm{O}$. This has led us to predict two different stable stoichiometries, $\mathrm{HeH}_{2} \mathrm{O}$ and $\mathrm{He}_{2} \mathrm{H}_{2} \mathrm{O}$, which have lower formation enthalpies than mixtures of $\mathrm{H}_{2} \mathrm{O}$ and $\mathrm{He}$ at 2-8 and 8-92 $\mathrm{GPa}$, respectively, as shown in the convex hulls and the phase diagram of the pressure-components in Fig. 1 (a) and (b). These new phases adopt the tetragonal $I 4_{1} m d$ $\mathrm{HeH}_{2} \mathrm{O}$ structure and the cubic $F d \overline{3} m \mathrm{He}_{2} \mathrm{H}_{2} \mathrm{O}$ structures, the symmetry of the system changes from $I 4_{1} m d$ to $\mathrm{Fd} \overline{3} \mathrm{~m}$ at around $55 \mathrm{GPa}$. At low pressures the $\mathrm{HeH}_{2} \mathrm{O}$ and $\mathrm{He}\left(\mathrm{H}_{2} \mathrm{O}\right)_{2}$ compositions become energetically favorable. Our calculations suggest that the $I 4_{1}$ md $\mathrm{HeH}_{2} \mathrm{O}$ phase and the previously proposed clathrate structure $C m c 2_{1}$ phase $\mathrm{He}\left(\mathrm{H}_{2} \mathrm{O}\right)_{2}$ [37] are stable even close to ambient pressure. We also note that vdW effects play a dominant role in the stability of $\mathrm{He}^{-} \mathrm{H}_{2} \mathrm{O}$ compounds under pressure, and the effects of volume reduction is also a key element, especially in $\mathrm{He}_{2} \mathrm{H}_{2} \mathrm{O}$.

Our searches also provide other candidate structures that have enthalpies that are very close (less than 1 $\mathrm{meV} /$ atom) to the lowest enthalpy structures presented in the main text. For instance, the energy of $P 4_{3} 2_{1} 2$ $\mathrm{HeH}_{2} \mathrm{O}$ is very close to that of $I 4_{1} m d \mathrm{HeH}_{2} \mathrm{O}$; the $C m c 2_{1} \mathrm{He}_{2} \mathrm{H}_{2} \mathrm{O}$ structure has a similar enthalpy to that of $I 4_{1} m d \mathrm{He}_{2} \mathrm{H}_{2} \mathrm{O}$; while the enthalpy of a $P 6_{3} / m m c$ $\mathrm{He}_{2} \mathrm{H}_{2} \mathrm{O}$ phase is similar to that of $F d \overline{3} m \mathrm{He}_{2} \mathrm{H}_{2} \mathrm{O}$. The relevant structures are listed in Table I in the Supplemen-

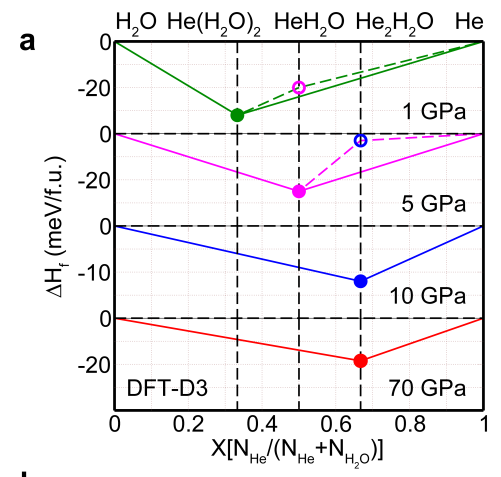

c



b

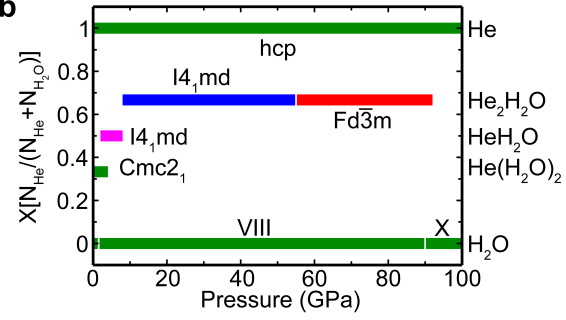

d
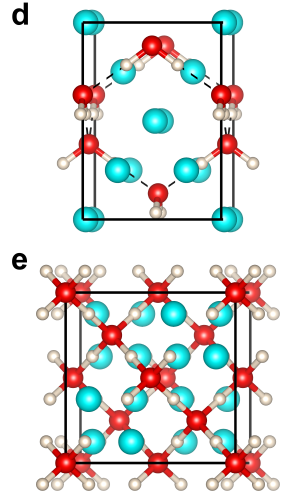

FIG. 1. Thermodynamics of the $\mathrm{He}^{-} \mathrm{H}_{2} \mathrm{O}$ system and crystal structures of the stable compounds. (a) Convex hull for formation enthalpies $\left(\Delta H_{f}\right.$, with respect to $\mathrm{He}$ and $\left.\mathrm{H}_{2} \mathrm{O}\right)$ at different pressures, calculated with the DFT-D3 functional. (b) Pressure-composition phase diagram of the He- $\mathrm{H}_{2} \mathrm{O}$ phases (magenta, blue and red lines) discovered in this work, together with the previously known phases (green lines) below $100 \mathrm{GPa}$. (c-e) Crystal structures of three new phases: (c) $I 4_{1} m d \mathrm{HeH}_{2} \mathrm{O}$, (d) $I 4_{1} m d \mathrm{He}_{2} \mathrm{H}_{2} \mathrm{O}$ and (e) $F d \overline{3} m$ $\mathrm{He}_{2} \mathrm{H}_{2} \mathrm{O}$.

tary Information. It is interesting that the ice framework of $P 6_{3} / m m c \mathrm{He}_{2} \mathrm{H}_{2} \mathrm{O}$ is close to that of hexagonal ice, while the ice framework in $F d \overline{3} m \mathrm{He}_{2} \mathrm{H}_{2} \mathrm{O}$ appears similar to cubic ice.

As shown in Fig. 1 (d) and (e), the $\mathrm{H}_{2} \mathrm{O}$ molecule sublattice in $I 4_{1} m d$ and $F d \overline{3} m \mathrm{He}_{2} \mathrm{H}_{2} \mathrm{O}$ are similar to those of ice VIII and ice X, respectively. As recently shown by Bronstein et al.[38], $\mathrm{O}-\mathrm{H}-\mathrm{O}$ bond symmetrization occurs at around 90-100 GPa when the protons are considered as classical particles, and 60-65 GPa for quantum protons. Therefore the presence of helium significantly decreases the pressure at which hydrogen-bond symmetrization occurs in the helium-water system.

Diffusion of atoms. To study the dynamical properties of the predicted water-helium compounds we have performed extensive ab initio molecular dynamics $(A I M D)$ simulations within the pressure range 10-120 GPa and the temperature range 200-2600 K. Diffusion coefficients were calculated for the oxygen, hydrogen and helium atoms from their mean-square displacements (MSD). Superionic water is usually classified as one of three phases in terms of the diffusion coefficient of the $\mathrm{H}$ and $\mathrm{O}$ atoms: the solid phase $\left(D_{O}=0\right.$ and $\left.D_{H}=0\right)$, the superionic $(S I)$ phase $\left(D_{O}=0\right.$ and $\left.D_{H}>0\right)$ and the fluid phase 



FIG. 2. Behavior of $\mathbf{H}$ (green) and $\mathbf{H e}$ atoms (blue) compared to $\mathbf{O}$ atoms (red) in $F d \overline{3} m \mathbf{H e}_{2} \mathbf{H}_{2} \mathrm{O}$ from $A I M D$ simulations at $1600 \mathrm{~K}, 2000 \mathrm{~K}$ and $2300 \mathrm{~K}$. (a-c) The averaged mean squared displacements (MSD) for the H, He and $\mathrm{O}$ atoms from $A I M D$ simulations at different temperatures. (d-i) Representation of atomic trajectories in one supercell from the simulations from the last 5 ps run representing the three distinct phases: the solid phase (1600 K), the superionic He phase (2000 K), SI-I, and superionic He and H phase (2300 K), SI-II.

$\left(D_{O}>0\right.$ and $\left.D_{H}>0\right)$. Surprisingly, in our hydrated helium system we found a region of superionicity with a new diffusive helium phase $\left(D_{O}=0, D_{H}=0\right.$ and $\left.D_{H e}>0\right)$ that we have named as $S I$-I, and a coexisting diffusive helium and hydrogen phase $\left(D_{O}=0, D_{H}>0\right.$ and $D_{H e}>0$ ) named $S I$-II, as shown in Fig. 2 (a-c). We have also confirmed our results for the radial distribution functions of the averaged structures, as shown in Supplementary Fig. S1. To confirm the superionic features we have distinguished the two superionic phases (SI-I and $S I$-II) by comparing their atomic trajectories from sim- ulations at around $90 \mathrm{GPa}$, as shown in Fig. 2 (d-i).

The Hydrogen and Helium trajectories overlap with one another, and for clarity we show them in separate plots. Therefore in Fig. 2 (d-f) only the oxygen and hydrogen atoms are plotted, while in Fig. 2 (g-i), only the oxygen and helium atoms are plotted. At around $90 \mathrm{GPa}$ and a starting temperature below $1600 \mathrm{~K}$, the atoms are tethered at their starting positions and vibrate around them. From the diffusion constants of the atoms ( $D_{O}=0, D_{H}=0$ and $\left.D_{H e}=0\right)$ one finds that helium hydride maintains the solid phase under these conditions. 
However, when the temperature is increased to 2000 $\mathrm{K}$, although the oxygen and hydrogen atoms vibrate on their original sublattices, the helium atoms vibrate much more strongly and even jump to neighboring sites which results in a non-zero diffusion constant $D_{H e}=0.14 \times 10^{-8}$ $\mathrm{m}^{2} / \mathrm{s}$. At $2300 \mathrm{~K}$ both hydrogen and helium atoms are delocalized from their initial sites and their diffusion coefficients are both non-zero $\left(D_{H}=0.21 \times 10^{-8} \mathrm{~m}^{2} / \mathrm{s}\right.$ and $\left.D_{H e}=0.33 \times 10^{-8} \mathrm{~m}^{2} / \mathrm{s}\right)$. The diffusion coefficients calculated from the velocity autocorrelation functions are very similar to those obtained from the MSD, as shown in Table II of the Supplementary Information. Both helium and hydrogen atoms have larger diffusivities and therefore larger probabilities of moving into the interstitial spaces of the oxygen sublattice. Finally, increasing the temperature beyond $2400 \mathrm{~K}$ leads to melting of the helium hydrate and the atoms diffuse freely (not shown here).

Our simulations show that the three atomic species in helium hydrate: oxygen, hydrogen and helium, have different "melting" temperatures above which diffusion occurs $T_{H e}<T_{H}<T_{O}$. The law of equipartition states that in equilibrium the energy should be equally distributed among the degrees of freedom. Therefore with the same kinetic energy, the lighter atoms should move faster in non-interacting systems. Within our helium-water system the helium atoms have a higher mobility and a lower "melting" temperature than hydrogen atoms, although helium is heavier than hydrogen.

To investigate the reasons for this abnormal diffusive behavior we calculated the electron localization function (ELF) of the hydrate $F d \overline{3} m$ phase at $0 \mathrm{~K}$, as shown in Supplementary Fig. S2. We plotted an ELF isosurface (isovalue $=0.7$ ) in the conventional unit cell and a crosssection along the $\langle 011\rangle$ plane. This demonstrates that electrons localize along the bonds between the oxygen and hydrogen atoms and are isolated in the helium atoms. This shows that the $\mathrm{O}-\mathrm{H}$ interactions arise from strong covalent bonds while the helium atoms have weak vdW interactions with the $\mathrm{H}_{2} \mathrm{O}$ framework. This results in a higher diffusion barrier for hydrogen atoms than for helium in the finite temperature superionic phases.

Phase diagram. Inspired by the temperature-induced features in helium hydrate discussed above, we expanded the pressure range studied to explore the superionic region from ambient pressure up to about $100 \mathrm{GPa}$ with pressure steps of $20 \mathrm{GPa}$. In superionic water the two different "melting" temperatures of hydrogen and oxygen in ice divide its phase diagram into three regions: solid, superionic, and liquid. As shown in Fig. 3, the three "melting" temperatures of helium, hydrogen and oxygen divide the phase diagram of helium hydrate into four distinct regions: the solid, superionic helium (SI-I), superionic helium + hydrogen $(S I-\mathrm{II})$, and fluid phases. As expected, the superionicity is enhanced with increasing



FIG. 3. Proposed phase diagram of the helium-water system at high pressures obtained from our structure searches and $A I M D$ simulations. Symbols represent four distinct thermodynamic states sampled in our simulations: circle, solid state; square, He diffusive state $(S I-I)$; diamond, both $\mathrm{He}$ and $\mathrm{H}$ diffusive state (SI-II); and triangle, fluid state. Black dashed lines were fitted to the phase transition boundaries. Red dashed lines distinguish the two predicted solid phases: $I 4_{1} m d$ and $F d \overline{3} m$, as well as two types of $\mathrm{H}_{2} \mathrm{O}$ sublattice $\left(I 4_{1} m d\right.$ and $\left.F d \overline{3} m\right)$ in the $S I$-I region. Isentropes for Uranus and Neptune (Dark green and blue solid lines) and phase boundary for superionic pure water (white dash-dotted line) are taken from Ref. [8].

pressure and the superionic helium region $(S I-\mathrm{I})$ exists over the entire pressure range studied, while the superionic helium + hydrogen phase $(S I$-II) is stable only above $40 \mathrm{GPa}$. The pressure-induced transition from $I 4_{1} m d$ to $F d \overline{3} \mathrm{~m}$ is in fact a continuous second-order phase transition, as shown in Supplementary Fig. S3. The temperature may therefore have a critical influence on the transition pressure between these two phases at high pressure.

Sun et al. [12] recently reported that the sublattice of the oxygen atoms in superionic water shows a complicated sequence of phase transitions. Examination of the trajectories in the superionic region obtained by averaging atomic positions shows that the oxygen sublattices in both $S I$-I and $S I$-II retain an FCC arrangement in helium hydrate, which has the same oxygen sublattices in solid $I 4_{1} m d$ and $F d \overline{3} \mathrm{~m} \mathrm{He}_{2} \mathrm{H}_{2} \mathrm{O}$. Moreover, we have calculated radial distribution functions for these averaged structures and compared them with the initial zero-temperature structure. At $30 \mathrm{GPa}$ the $\mathrm{H}_{2} \mathrm{O}$ sublattice maintains its original positions as in the $I 4_{1} m d$ phase at low temperatures and then transforms into a sublattice of the $F d \overline{3} m$ phase with increasing temperature, as shown in Supplementary Fig. S4.

\section{Discussion}

We have also studied superionicity in the low pressure phase by gradually increasing the temperature in 


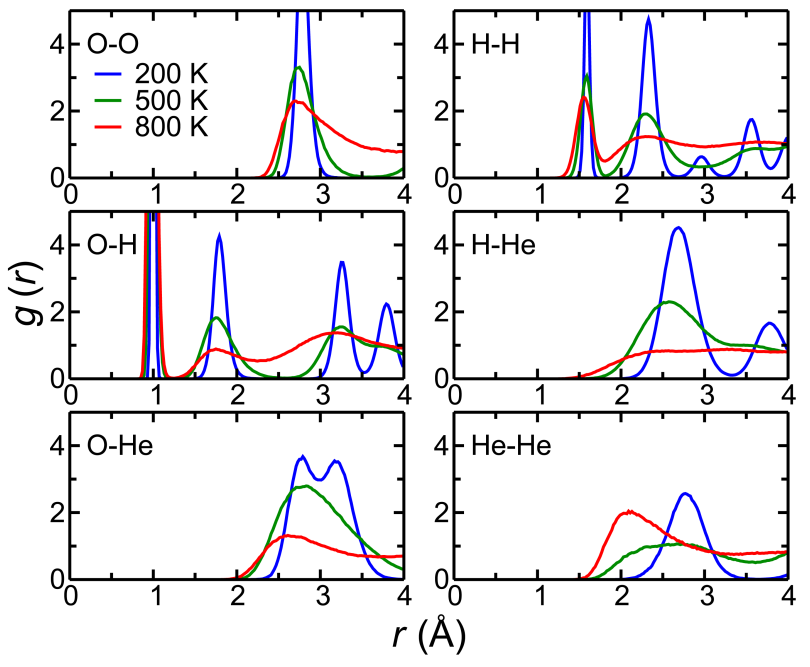

FIG. 4. Radial distribution functions $\mathbf{g}(\mathbf{r})$ of the $I 4_{1} m d$ $\mathbf{H e H}_{2} \mathbf{O}$ phase. The $A I M D$ simulations are performed at around $3 \mathrm{GPa}$ and heating to around $200 \mathrm{~K}$ (blue lines), 500 $\mathrm{K}$ (green lines) and $800 \mathrm{~K}$ (red lines), corresponding to the solid phase, diffusive helium phase $(S I-\mathrm{I})$ and fluid phase, respectively.

the $A I M D$ simulations. The RDFs in Fig. 4 obtained from simulations at three different temperatures clearly indicate the presence of three states: the solid at 200 $\mathrm{K}$, superionic helium at $500 \mathrm{~K}$, and a fluid phase at 800 $\mathrm{K}$. $\mathrm{HeH}_{2} \mathrm{O}$ maintains its crystalline form at $200 \mathrm{~K}$, and almost all of the atomic pairs maintain their isolated peaks in the RDF, except for the combination of the first two peaks in the oxygen-helium RDF which exhibit large thermal fluctuations. The thermal fluctuations increase rapidly with heating and the peaks in the oxygenoxygen, oxygen-hydrogen, and hydrogen-hydrogen RDFs are slightly broadened, but still show the characteristic peaks of a sublattice of $\mathrm{H}_{2} \mathrm{O}$ undergoing large amplitude vibrations.

In the superionic helium $(S I-\mathrm{I})$ phase at $500 \mathrm{~K}$ the thermal fluctuations increase rapidly and the peaks in the oxygen-oxygen, oxygen-hydrogen, and hydrogenhydrogen RDFs are slightly broadened, but they still show the characteristic peaks of a sublattice of $\mathrm{H}_{2} \mathrm{O}$ undergoing large amplitude vibrations. The RDF involving helium atoms shows only one maximum and then converges to a constant intensity, which represents the behavior of a liquid or a glass. Finally, at $800 \mathrm{~K}$ in the fluid phase the $\mathrm{H}_{2} \mathrm{O}$ sublattice distorts from the hydrogenhydrogen RDF. We find that the first peak still exists at the same position even in the fluid, and the O-H distance in the superionic state remains similar to that in ice.

Previous work [14] shows that the quantum correction to the internal energy in MD simulations is affected by the pressure and temperature in water ice. Our results

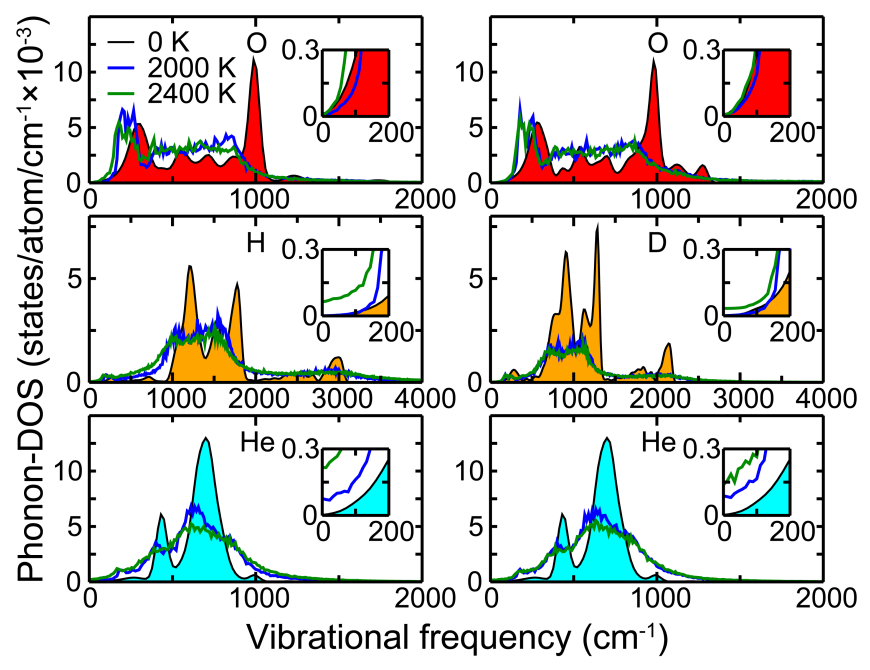

FIG. 5. Vibrational density of states of $F d \overline{3} m$ phase $\mathrm{He}_{2} \mathrm{H}_{2} \mathrm{O}$ (left) and $\mathrm{He}_{2} \mathrm{D}_{2} \mathrm{O}$ (right). Colored areas under black curves give the Phonon-DOS of O (red), H/D (orange) and $\mathrm{He}$ (cyan) from static phonon calculations at 0 $\mathrm{K}$. The blue and dark green lines represent the vibrational DOS of each atomic species in the $S I$-I phase at $2000 \mathrm{~K}$ and $S I$-II phase at $2400 \mathrm{~K}$, respectively, calculated from the Fourier transform of the velocity autocorrelation function. The low frequency regions are enlarged in the insets and the zero/non-zero vibrational DOS at zero frequency shows the fixed/diffusive behavior of $\mathrm{O}, \mathrm{H} / \mathrm{D}$, and $\mathrm{He}$ at different temperatures.

(Supplementary Table II) show that the nuclear quantum corrections $\mathrm{u}_{q c}$ for helium-water compounds are on a similar scale to those found in the literature for ice [14]. This is relatively small compared with the internal energy $\mathrm{u}_{m d}$, and therefore it does not greatly affect the occurance of superionic phases in helium-water compounds.

In addition, Hermann et al. [39] studied zero-point energies of water ice at megabar pressures by replacing hydrogen atoms with its isotopes, deuterium and tritium. The isotope effect can also emerge in the dynamical properties such as the diffusion coefficient and vibrational spectra. Here, we estimate the influence of nuclear quantum effects in the $S I$-I phase (at $2000 \mathrm{~K}$ ) and $S I$-II phase (at $2400 \mathrm{~K}$ ) by comparing the vibrational density of states in $\mathrm{He}_{2} \mathrm{H}_{2} \mathrm{O}$ and $\mathrm{He}_{2} \mathrm{D}_{2} \mathrm{O}$. As shown in Fig. 5, with increasing mass, in the zero temperature phonon DOS, the high frequency phonon peak decreases from about $3000 \mathrm{~cm}^{-1}$ for hydrogen, to about $2100 \mathrm{~cm}^{-1}$ for deuterium. At $2000 \mathrm{~K}$, both $\mathrm{H}$ and $\mathrm{D}$ are fixed in their sublattices and have very similar vibrational frequencies to their corresponding zero temperature phonon frequencies. As shown in the insets, He atoms have a non-zero diffusive coefficient at zero frequency, which represents the $S I$-I phase. At $2400 \mathrm{~K}$, the $\mathrm{H}$ and D, as well as the He atoms have non-zero diffusive coefficients at zero frequency, while only the oxygen atoms are fixed, which cor- 
responds to the $S I$-II phase. These results indicate that nuclear quantum effects do not significantly affect the existance of the superionic states in helium-water compounds.

In summary, we have used a combination of $a b i n i$ tio crystal structure searches and AIMD simulations based on DFT to perform our calculations. We have systematically explored the helium-water phase diagram below $100 \mathrm{GPa}$ and found three thermodynamically stable solid phases: the tetragonal $I 4_{1}$ md $\mathrm{HeH}_{2} \mathrm{O}$, the $I 4_{1}$ md $\mathrm{He}_{2} \mathrm{H}_{2} \mathrm{O}$ and the cubic $F d \overline{3} m \mathrm{He}_{2} \mathrm{H}_{2} \mathrm{O}$ phases. The pressure at which hydrogen-bond symmetrization occurs is reduced to around $55 \mathrm{GPa}$ by introducing helium. More interestingly, we find a superionic helium region in the helium hydrate phase diagram with highly mobile helium atoms and a fixed sublattice of ice at relatively low temperatures. Further heating leads to a superionic phase in which the motions of hydrogen and helium atoms give rise to superionicity, while the oxygen atoms vibrate around the original sublattice. These three atomic types in the helium hydrate exhibit abnormal "melting" properties $\left(T_{H e}<T_{H}<T_{O}\right)$ marked by a change in diffusion rates, molecular dynamics trajectories and radial distribution functions. These properties divide the phase diagram into four distinguishable regions: solid, superionic helium, superionic helium + hydrogen and fluid. At relatively low pressures (below $40 \mathrm{GPa}$ ), the superionic helium + hydrogen region is absent and the superionic helium region remains close to ambient pressure. The insertion of helium substantially reduces the pressures at which superionic states can be formed compared to pure ice which may be more easily accessed in future experiments. Although the pressures and temperatures in this work are somewhat lower than those in the isentropes of Uranus or Neptune, the insertion of helium could alter the superionicity of pure water, which may still exist in Uranus or Neptune. Meanwhile, as the second most abundant element, helium may exist in planets or moons with different masses, compositions and distances from their own main stars. These planets and moons may have different isentropic conditions which could allow the formation of superionic $\mathrm{He}-\mathrm{H}_{2} \mathrm{O}$ compounds.

\section{Online content}

Any methods, additional references, Nature Research reporting summaries, source data, statements of code and data availability and associated accession codes are available at https://xxx.xxx-xxxxx

[1] Demontis, P., LeSar, R. \& Klein, M. L. New HighPressure Phases of Ice. Phys. Rev. Lett. 60, 2284-2287 (1988).

[2] Cavazzoni, C. et al. Superionic and Metallic States of Water and Ammonia at Giant Planet Conditions. Science
283, 44-46 (1999).

[3] Yakushev, V., Postnov, V., Fortov, V. \& Yakysheva, T. Electrical conductivity of water during quasi-isentropic compression to $130 \mathrm{GPa}$. J. Exp. Theor. Phys. 90, 617622 (2000).

[4] Chau, R., Mitchell, A., Minich, R. \& Nellis, W. Electrical conductivity of water compressed dynamically to pressures of 70-180 GPa (0.7-1.8 Mbar). J. Chem. Phys. 114, 1361-1365 (2001).

[5] Goncharov, A. F. et al. Dynamic Ionization of Water under Extreme Conditions. Phys. Rev. Lett. 94, 125508 (2005).

[6] Goldman, N., Fried, L. E., Kuo, I.-F. W. \& Mundy, C. J. Bonding in the Superionic Phase of Water. Phys. Rev. Lett. 94, 217801 (2005).

[7] French, M., Mattsson, T. R., Nettelmann, N. \& Redmer, R. Equation of state and phase diagram of water at ultrahigh pressures as in planetary interiors. Phys. Rev. B 79, 054107 (2009).

[8] Redmer, R., Mattsson, T. R., Nettelmann, N. \& French, M. The phase diagram of water and the magnetic fields of Uranus and Neptune. Icarus 211, 798-803 (2011).

[9] Ninet, S., Datchi, F. \& Saitta, A. M. Proton Disorder and Superionicity in Hot Dense Ammonia Ice. Phys. Rev. Lett. 108, 165702 (2012).

[10] Sugimura, E. et al. Experimental evidence of superionic conduction in $\mathrm{H}_{2} \mathrm{O}$ ice. J. Chem. Phys. 137, 194505 (2012).

[11] Wilson, H. F., Wong, M. L. \& Militzer, B. Superionic to Superionic Phase Change in Water: Consequences for the Interiors of Uranus and Neptune. Phys. Rev. Lett. 110, 151102 (2013).

[12] Sun, J., Clark, B. K., Torquato, S. \& Car, R. The phase diagram of high-pressure superionic ice. Nature Commun. 6, 8156 (2015).

[13] Bethkenhagen, M., Cebulla, D., Redmer, R. \& Hamel, S. Superionic Phases of the 1:1 WaterAmmonia Mixture. J. Phys. Chem. A 119, 10582-10588 (2015).

[14] French, M., Desjarlais, M. P. \& Redmer, R. Ab initio calculation of thermodynamic potentials and entropies for superionic water. Phys. Rev. E 93, 022140 (2016).

[15] Hernandez, J.-A. \& Caracas, R. Superionic phase transitions in Body-Centered Cubic $\mathrm{H}_{2} \mathrm{O}$ ice. Phys. Rev. Lett. 117, 135503 (2016).

[16] Bethkenhagen, M. et al. Planetary Ices and the Linear Mixing Approximation. Astrophys. J. 848, 67 (2017).

[17] Jiang, X., Wu, X., Zheng, Z., Huang, Y. \& Zhao, J. Ionic and superionic phases in ammonia dihydrate $\mathrm{NH}_{3} \cdot \mathrm{H}_{2} \mathrm{O}$ under high pressure. Phys. Rev. B 95, 144104 (2017).

[18] Hernandez, J.-A. \& Caracas, R. Proton dynamics and the phase diagram of dense water ice. J. Chem. Phys. 148, 214501 (2018).

[19] Millot, M. et al. Experimental evidence for superionic water ice using shock compression. Nature Phys. 14, 297 (2018).

[20] Millot, M. et al. Nanosecond X-ray diffraction of shockcompressed superionic water ice. Nature 569, 251-255 (2019).

[21] Kamaya, N. et al. A lithium superionic conductor. Nature Mater. 10, 682-686 (2011).

[22] Wang, X., Xiao, R., Li, H. \& Chen, L. Oxysulfide LiAlSO: A Lithium Superionic Conductor from First Principles. Phys. Rev. Lett. 118, 195901 (2017).

[23] He, X., Zhu, Y. \& Mo, Y. Origin of fast ion diffusion 
in super-ionic conductors. Nature Commun. 8, 15893 (2017).

[24] Liu, H. et al. Copper ion liquid-like thermoelectrics. $\mathrm{Na}$ ture Mater. 11, 422 (2012).

[25] Qiu, W. et al. Part-crystalline part-liquid state and rattling-like thermal damping in materials with chemicalbond hierarchy. Proc. Natl Acad. Sci. USA 111, 1503115035 (2014).

[26] Cazorla, C., Errandonea, D. \& Sola, E. High-pressure phases, vibrational properties, and electronic structure of $\mathrm{NeHe}_{2}$ and $\mathrm{ArHe}_{2}$ : A first-principles study. Phys. Rev. $B$ 80, 064105 (2009).

[27] Wang, Y., Zhang, J., Liu, H. \& Yang, G. Prediction of the XeHe binary phase diagram at high pressures. Chem. Phys. Lett. 640, 115-118 (2015).

[28] Vos, W. L. et al. A high-pressure van der Waals compound in solid nitrogen-helium mixtures. Nature $\mathbf{3 5 8}$, 46 (1992).

[29] Ninet, S., Weck, G., Loubeyre, P. \& Datchi, F. Structural and vibrational properties of the van der waals compound $\left(\mathrm{N}_{2}\right)_{11} \mathrm{He}$ up to $135 \mathrm{GPa}$. Phys. Rev. B 83, 134107 (2011).

[30] Dong, X. et al. A stable compound of helium and sodium at high pressure. Nature Chem. 9, 440-445 (2017).

[31] Monserrat, B., Martinez-Canales, M., Needs, R. J. \& Pickard, C. J. Helium-Iron Compounds at Terapascal Pressures. Phys. Rev. Lett. 121, 015301 (2018).

[32] Zhang, J. et al. Rare Helium-Bearing Compound $\mathrm{FeO}_{2} \mathrm{He}$ Stabilized at Deep-Earth Conditions. Phys. Rev. Lett. 121, 255703 (2018).

[33] Gao, H., Sun, J., Pickard, C. J. \& Needs, R. J. Prediction of pressure-induced stabilization of noble-gas-atom compounds with alkali oxides and alkali sulfides. Phys. Rev. Materials 3, 015002 (2019).

[34] Liu, Z. et al. Reactivity of He with ionic compounds under high pressure. Nature Commun. 9, 951 (2018).

[35] Li, Y. et al. Route to high-energy density polymeric nitrogen $t-\mathrm{N}$ via He-N compounds. Nature Commun. 9, 722 (2018).

[36] Liu, H., Yao, Y. \& Klug, D. D. Stable structures of He and $\mathrm{H}_{2} \mathrm{O}$ at high pressure. Phys. Rev. B 91, 014102 (2015).

[37] Teeratchanan, P. \& Hermann, A. Computational phase diagrams of noble gas hydrates under pressure. J. Chem. Phys. 143, 154507 (2015).

[38] Bronstein, Y., Depondt, P., Finocchi, F. \& Saitta, A. M. Quantum-driven phase transition in ice described via an efficient Langevin approach. Phys. Rev. B 89, 214101 (2014).

[39] Hermann, A., Ashcroft, N. W. \& Hoffmann, R. Isotopic differentiation and sublattice melting in dense dynamic ice. Phys. Rev. B 88, 214113 (2013).

\section{Acknowledgments}

J.S. gratefully acknowledges financial support from the MOST of China (Grant Nos. 2016YFA0300404, 2015CB921202), the National Natural Science Foundation of China (Grant Nos. 11574133 and 11834006), the NSF of Jiangsu Province (Grant No. BK20150012), the Science Challenge Project (No. TZ2016001), the Fundamental Research Funds for the Central Universities and Special Program for Applied Research on Su- per Computation of the NSFC-Guangdong Joint Fund (the 2nd phase) under Grant No.U1501501. C.J.P. and R.J.N. acknowledge financial support from the Engineering and Physical Sciences Research Council (EPSRC) of the U.K. under grants [EP/G007489/2] (C.J.P.) and [EP/P034616/1] (R.J.N.). C.J.P. also acknowledges financial support from EPSRC and the Royal Society through a Royal Society Wolfson Research Merit award. The calculations were carried out using supercomputers at high performance supercomputing center of Nanjing University, "Tianhe-2" at NSCC-Guangzhou and the CSD3 Peta4 CPU/KNL machine in the University of Cambridge.

\section{Authors contribution}

J.S. conceived the project. J.S. and H.T.W. led the project. C.L., H.G., Y.W. C.J.P performed the calculations. C.L., H.G., C.J.P and J.S. analyze the data. C.L., J.S., R.J.N, H.T.W. and D.X. wrote the manucript. All authors discussed the results and commented on the manuscript.

C. L. and H. G. contributed equally to this work.

Competing financial interests. CJP is an author of the CASTEP code, and receives royalty payments from its commercial sales by Dassualt Systemes.

\section{Additional information}

Supplementary Information accompanies this paper is available at http://xxx.xxx.xxx

Reprints and permissions information is available at www.nature.com/reprints.

Correspondence and requests for materials should be addressed to J.S. or H.T.W.

Publishers note: Springer Nature remains neutral with regard to jurisdictional claims in published maps and institutional affiliations. 


\section{Method}

We performed variable-composition structure predictions implemented in a machine-learning accelerated crystal structure search method[1]. The crystal structures obtained were cross-checked with results from the $a b$ initio random structure searching approach (AIRSS) $[2,3]$, which gave very similar results. The density functional theory (DFT) calculations were performed using the Vienna ab initio simulation package (VASP) [4] and the projector augmented-wave method and the generalized gradient approximation (GGA) and the PerdewBurke-Ernzerhof exchange correlation functional (PBE). [5]. We employed a plane wave cutoff energy of $720 \mathrm{eV}$ and a dense Monkhorst-Pack Brillouin zone integration grid with a resolution of $2 \pi \times 0.025 \AA^{-1}$. The relaxed ionic positions and cell parameters provided forces that are smaller than $10^{-5} \mathrm{eV}$ and $\mathrm{eV} / \AA$, respectively. The CASTEP code was used for the AIRSS searches, similar convergence parameters as used in VASP were employed [6]. To account for the vdW interactions we used the DFT-D3 [7], optB88-vdW [8] and rev-vdW-DF2 [9] functionals to cross check the quality of the energy minimization and the calculated zero-point energy. The results obtained with different functionals are very similar, see Supplementary Fig. S5. Phonon calculations show that the newly predicted structures are dynamically stable, as can be seen in Supplementary Fig. S6. Phonon spectra of the $I 4_{1} m d \mathrm{HeH}_{2} \mathrm{O}$ phases at $0 \mathrm{GPa}$ do not exhibit imaginary vibrational frequencies, which indicates that it may be quenchable even to ambient pressure. Results for the zero point energy (ZPE), finite temperature Gibbs free energy, and tests of the dynamical stability of the $\mathrm{He}-\mathrm{H}_{2} \mathrm{O}$ compounds are available in Fig. S7 and S8 in the Supplementary Information. Phonon calculations were performed using the phonopy package [10]. $A b$ initio molecular dynamics $(A I M D)$ simulations were performed in $4 \times 4 \times 4$ supercells (with 256 atoms for $\mathrm{HeH}_{2} \mathrm{O}$ and 320 atoms for $\mathrm{He}_{2} \mathrm{H}_{2} \mathrm{O}$, respectively) with $\Gamma$-centred $k$-point sampling. The results of convergence tests for the $k$-point mesh and the finite size effects are shown in Table III of the Supplementary Information. A
Nose-Hoover thermostat was used to perform the NVT simulations. Runs with 7000 steps were carried out with a time step of $1 \mathrm{fs}$, the initial 2 ps were used for thermalization and the final 5000 steps of each trajectory were used to extract the statistical quantities. Some trajectories were extended to $12 \mathrm{ps}$ to check the stability of the simulations.

\section{Data Availability}

The data that support the plots within this paper and other findings of this study are available from the corresponding authors upon reasonable request.

[1] Xia, K. et al. A novel superhard tungsten nitride predicted by machine-learning accelerated crystal structure search. Science Bulletin 63, 817-824 (2018).

[2] Pickard, C. J. \& Needs, R. J. High-Pressure Phases of Silane. Phys. Rev. Lett. 97, 045504 (2006).

[3] Pickard, C. J. \& Needs, R. J. Ab initiorandom structure searching. J. Phys.: Condens. Matter 23, 053201 (2011).

[4] Kresse, G. \& Furthmller, J. Efficient iterative schemes for ab initio total-energy calculations using a plane-wave basis set. Phys. Rev. B 54, 11169-11186 (1996).

[5] Perdew, J. P. et al. Atoms, molecules, solids, and surfaces: Applications of the generalized gradient approximation for exchange and correlation. Phys. Rev. B $\mathbf{4 6}$, 6671-6687 (1992).

[6] Clark, S. J. et al. First principles methods using CASTEP. Zeitschrift fr Kristallographie - Crystalline Materials 220, 567-570 (2009).

[7] Grimme, S., Antony, J., Ehrlich, S. \& Krieg, H. A consistent and accurate ab initio parametrization of density functional dispersion correction (DFT-D) for the 94 elements H-Pu. J. Chem. Phys. 132, 154104 (2010).

[8] Klime, J., Bowler, D. R. \& Michaelides, A. Chemical accuracy for the van der Waals density functional. $J$. Phys.: Condens. Matter 22, 022201 (2009).

[9] Hamada, I. van der Waals density functional made accurate. Phys. Rev. B 89, 121103 (2014).

[10] Togo, A. \& Tanaka, I. First principles phonon calculations in materials science. Scripta Materialia 108, 1-5 (2015). 